\title{
Anterograde and Retrograde Amnesia After Lesions to Frontal Cortex in Rats
}

\author{
Gordon Winocur ${ }^{1,2,3,4}$ and Morris Moscovitch ${ }^{1,3}$ \\ ${ }^{1}$ Rotman Research Institute, Baycrest Centre for Geriatric Care, Toronto, Ontario M6A 2E1, Canada, ${ }^{2}$ Department of \\ Psychology, Trent University, Peterborough, Ontario K9J 7B8, Canada, and Departments of ${ }^{3}$ Psychology and ${ }^{4}$ Psychiatry, \\ University of Toronto, Toronto, Ontario M5S 3G3, Canada
}

A socially acquired food-preference test was used to assess effects of lesions to the frontal cortex on anterograde and retrograde memory in rats. In Experiment 1, there was no effect of lesion when rats were administered a two-choice test in which the target food was to be selected in the presence of a single distractor. In Experiment 2, a three-choice memory test was administered in which the target food was presented along with two equally palatable alternatives. In the latter test, lesioned groups displayed anterograde amnesia that increased with the length of the interval between postoperative acquisi-

Investigations of memory function in humans and animals with frontal lobe damage have been concerned mainly with anterograde memory but, in recent years, there has been increased interest in retrograde memory (for review, see Moscovitch, 1994; Wheeler et al., 1995, 1997). In contrast to the temporally graded retrograde amnesia that has been reported in amnesic patients with medial temporal lobe-hippocampal lesions (Marslen-Wilson and Teuber, 1975; Rempel-Clower et al., 1996; Nadel and Moscovitch, 1997; Reed and Squire, 1998), frontal lobe patients typically exhibit a flat gradient in which memory loss extends equally over many years. The animal work has focused even more than the human studies on anterograde memory and, indeed, there appears not to have been a single study that examined the effects of lesions to frontal cortex on retrograde memory in animals.

The absence of retrograde memory studies in the animal literature is caused in part by the shortage of suitable tests that accurately measure memory for discrete events that occurred at specific times. In recent years, however, a number of promising tests have appeared (Zola-Morgan and Squire, 1990; Kim and Fanselow, 1992; Cho et al., 1995). One that has proven to be effective in measuring both anterograde and retrograde memory function in rats is based on a procedure devised originally by Galef and Wigmore (1983). In this test, which involves memory for an acquired food preference, a subject $(\mathrm{S})$ rat is paired with a demonstrator (D) rat that has recently eaten food with a distinctive taste. By interacting with D, S learns to prefer that food over other foods that have not been experienced in the same way. In

\footnotetext{
Received March 10, 1999; revised Aug. 12, 1999; accepted Aug. 16, 1999.

This work was supported by grants to G.W. and M.M. from the Natural Sciences and Engineering Research Council of Canada. The technical assistance provided by Chris Conley, John Zomer, Gordon Figuerro, and Heidi Roesler is also gratefully acknowledged.

Correspondence should be addressed to Dr. Gordon Winocur, Rotman Research Institute, Baycrest Centre for Geriatric Care, 3560 Bathurst Street, Toronto, Ontario M6A 2E1, Canada. E-mail: winocur@psych.utoronto.ca.

Copyright (C) 1999 Society for Neuroscience 0270-6474/99/199611-07\$05.00/0
}

tion and test, and a severe retrograde amnesia that extended equally over the entire range of intervals between preoperative acquisition and test. This outcome, which contrasted with the pattern of memory loss previously observed in rats with hippocampal lesions on this test, was interpreted as evidence for the strategic role of the frontal lobes in directing response selection and retrieval processes in memory.

Key words: anterograde amnesia; retrograde amnesia; foodpreference test; lesions; frontal cortex; rats

normal rats, the memory for an acquired food preference can last for 3-4 weeks.

This procedure was used to compare the effects of lesions to the hippocampus or dorsomedial thalamus on memory for food preferences that were acquired postoperatively (anterograde) or preoperatively (retrograde) (Winocur, 1990). All groups learned the preferences equally well in both conditions, but the lesioned groups displayed different patterns of memory performance. In the anterograde test, rats with hippocampal lesions forgot the preference faster than thalamus-damaged or normal rats (Munsey and Eichenbaum, 1995). In the retrograde test, hippocampal lesions produced a temporally graded retrograde amnesia (RA) that was similar to that seen in medial temporal lobe amnesics (Marslen-Wilson and Teuber, 1975; Rempel-Clower et al., 1996). By comparison, there were no meaningful differences between thalamic and control groups at any of the delay intervals in the anterograde and retrograde memory tests.

Winocur's (1990) study showed that the food-preference test was sensitive to the differential effects of selective brain lesions on anterograde and retrograde memory. Accordingly, the present research was conducted to study memory function in rats with lesions to the frontal cortex. A principal objective was to determine if the pattern of remote memory failure observed in patients with frontal lobe lesions was also evident in the rat model. It was of particular interest to know if a lesion-induced deficit was temporally graded, and this was achieved by assessing memory for food preferences acquired recently or at longer delays.

An enduring issue with respect to RA is its relation to anterograde amnesia (AA). There is evidence that the two can be closely related (Rempel-Clower et al., 1996; Reed and Squire, 1998) or quite independent of each other (Winocur et al., 1984; Shimamura and Squire, 1986; Kopelman, 1989;). This controversy is mirrored in neuropsychological studies of memory function in frontal lobe patients (Kopelman, 1989; Schmidtke and Vollmer, 1997). The food preference paradigm provides an op- 
portunity to address this issue in a rat model in which the effects of frontal cortex lesions are directly compared on anterograde and retrograde versions of the same memory test.

\section{EXPERIMENT 1}

The procedure developed for the Winocur (1990) study was followed in Experiment 1. In the test of RA, $\mathrm{S}$ rats acquired the food preference 10,5 , or $2 \mathrm{~d}$ before surgery. Ten days after surgery, they were presented with a choice of the sample food and a new food, and allowed to eat ad libitum for $2 \mathrm{hr}$. Several weeks later, the rats were administered the test of anterograde memory. For this test, memory for a newly acquired food preference was measured immediately, $2 \mathrm{~d}$, or $8 \mathrm{~d}$ after the S-D interaction.

\section{MATERIALS AND METHODS}

\section{Subjects and apparatus}

Thirty male, Long-Evans rats served as Ss in this experiment. An additional 10 rats served as Ds in the retrograde and anterograde memory tasks. The rats were obtained from the Trent University Breeding Center and were $\sim 8$ months old at the beginning of the experiment. Throughout the experiment, the rats were housed individually in standard wire cages $(25 \times 18 \mathrm{~cm}$. $)$ with food and water available ad libitum. Testing took place in larger cages $(42 \times 27 \times 24 \mathrm{~cm})$ divided into two equal compartments by a $1.25 \mathrm{~cm}$ wire mesh partition.

\section{Surgery and histology}

The $\mathrm{S}$ rats were assigned in equal numbers to frontal cortex (FC) and operated control (OC) groups. Group assignment was conducted semirandomly immediately after preoperative, food-preference training in the retrograde memory test. After surgical procedures, described previously (Winocur, 1992; Winocur and Eskes, 1998), rats were anesthetized with sodium pentobarbital $(50 \mathrm{mg} / \mathrm{kg}$ ) and positioned in a stereotaxic instrument. In the rats that received frontal cortex lesions, a midline incision was made, and $5 \mathrm{~mm}$ (in diameter) holes were drilled in the anterior skull. Frontal tissue was aspirated in the dorsolateral and medial regions of the structure through a 20 gauge hypodermic needle. The rats in the OC groups had holes drilled in their skulls, but no brain tissue was removed. After the experiment, rats in the FC group were deeply anesthetized with ether and perfused through the heart with physiological saline followed by $10 \%$ formol saline. The brains were embedded with paraffin before sectioning. All sections were stained with thionin, and the extent of damage was determined by comparisons with the standard atlas of König and Klippel (1963).

\section{Procedure}

Retrograde memory test. All D and S rats were placed on a $23.5 \mathrm{hr}$ food deprivation schedule for 1 week before being transferred to the test cages. The experimental procedure consisted of five discrete behavioral stages: (1) D and S rats were placed individually in separate compartments of test cages and left undisturbed for $2 \mathrm{~d}$ with ad libitum access to standard rat chow and water. This stage allowed the rats to become familiar with each other and their new environments. (2) The next day, food was removed from both cages. (3) After $23 \mathrm{hr}$ of food deprivation, the $\mathrm{D}$ rat was removed to another room and, for $60 \mathrm{~min}$, fed powdered rat chow mixed with commercially prepared cocoa ( $2 \%$ by weight) or commercially ground cinnamon ( $1 \%$ by weight). (4) Immediately thereafter, $\mathrm{D}$ was returned to its compartment and allowed to interact with $\mathrm{S}$ for 30 min through the wire mesh partition.

After stage 4, Ss were returned to their home cages for 2, 5, or $10 \mathrm{~d}$, during which they were fed 20 gm of standard rat chow in pellet form once each day. After the appropriate interval, rats were subjected to FC or OC surgery. After surgery, Ss were placed on ad libitum food for $5 \mathrm{~d}$, followed by a $23.5 \mathrm{hr}$ food deprivation schedule for 5 more days. The fifth, or test, stage of the behavioral procedure occurred for all rats at $10 \mathrm{~d}$ after surgery. This meant that $\mathrm{S}$ rats were tested 12,15 , or $20 \mathrm{~d}$ after they interacted with the D rats and acquired the food preference. For the test, $\mathrm{S}$ rats were returned individually to the test compartment where they were offered two food cups. One cup contained $30 \mathrm{gm}$ of the cocoaflavored diet, and the other contained $30 \mathrm{gm}$ of the cinnamon-flavored diet. The food cups were located in the corners of one end of the test cage. The locations of the sample and distractor diets were randomly varied between $\mathrm{S}$ rats. The Ss were allowed $2 \mathrm{hr}$ to eat from the food cups ad libitum, with water available at all times. The amount of food in the cups was weighed at 1 and $2 \mathrm{hr}$ intervals. The measure of a rat's preference for the sample diet was the amount of that diet consumed, expressed as a percentage of the total amount of food consumed. In a departure from the previous study (Winocur, 1990), a 0 delay condition, in which surgery was performed immediately after the D-S interaction, was not included. This was because the previous results showed that the general effect of invasive brain surgery, performed immediately after acquisition of the food preference, was sufficient to obliterate memory for preference, irrespective of the target site.

After the retrograde memory test, Ss were returned to their home cages and an ad libitum standard chow and water diet.

Anterograde memory test. Six to eight weeks elapsed during which the $\mathrm{S}$ rats participated in other experiments involving operant procedures. At this time, they were once again transferred individually to compartments of the test cage and allowed to interact with a new D in the other compartment (Stage 1). Stages 2-4 were identical to those followed in the retrograde memory test except that, in Stage 3, D and S rats interacted for only $15 \mathrm{~min}$ [this was done primarily to maintain consistency with the procedures followed by Winocur (1990)]. For the Stage 4 interaction, the sample food for each S rat was the food that served as the distractor in the retrograde memory test. That is, if an $\mathrm{S}$ rat had acquired a preference for the cocoa-flavored food in the retrograde memory test, this time it interacted with a D rat that was fed the cinnamon-flavored food, and vice versa. This procedure necessarily meant that, for the anterograde memory test, the rats were familiar with both diets. This was not considered a factor that would affect the results for several reasons. Memory for an acquired food preference in this paradigm is time-limited, lasting no longer than a few weeks and certainly less than the time between RA and AA testing. Moreover, a rat's preference at the choice test depends on memory for the most recently sampled food and not on general familiarity. As reported below in the Results section, control rats in the present study performed similarly to control rats in the Winocur (1990) study, where different groups were used in the retrograde and anterograde memory tests. Finally, as also reported below, the deficits of the frontal cortex-lesioned rats were related to factors other than familiarity of the diets.

In Stage 5, D was removed from the cage and, in the 0 delay condition, each S was offered two food-cups, one containing $30 \mathrm{gm}$ of the cocoaflavored diet and the other, $30 \mathrm{gm}$ of the cinnamon-flavored diet. The $\mathrm{S}$ rat was allowed to eat from the two food cups ad libitum, with water available throughout the session. The amount of food eaten from each cup was weighed at 1 and $2 \mathrm{hr}$ intervals. The measure of a rat's preference for the sample diet was the amount of that diet consumed, expressed as a percentage of the total amount of food consumed. Other S rats were tested at delays of 2 or $8 \mathrm{~d}$. During the delays, they were returned to their home cages and fed $20 \mathrm{gm}$ of standard rat chow in pellet form each day. After the delay, S rats were returned individually to the test compartment and allowed to eat for $2 \mathrm{hr}$ from the cinnamon- and cocoa-flavored diets in the usual manner. $\mathrm{S}$ rats were assigned to the various delay conditions on a semirandom basis to ensure equal numbers in all groups.

\section{RESULTS}

Examination of the food-preference measures indicated that neither FC nor OC groups had a bias for either the cinnamon or cocoa diet. ANOVA, conducted to compare the effect of diet on test performance, did not reveal significant effects of diet or significant group $\times$ diet interactions in either the retrograde memory or anterograde memory tests (all $p$ values $>0.05$ ). Accordingly, the data for each diet were combined and are presented as the sample diet eaten by $\mathrm{S}$ rats, as a percentage of the total amount of food consumed at each delay period.

In the food-preference tests, no significant group differences were noted between the 1 and $2 \mathrm{hr}$ measures at any of the delays in the anterograde and retrograde memory tasks. The only notable variation was that groups generally reduced their food intake in the second hour. Accordingly, only the results of the $2 \mathrm{hr}$ measure, which reflected the total amount of food consumed, will be reported. 


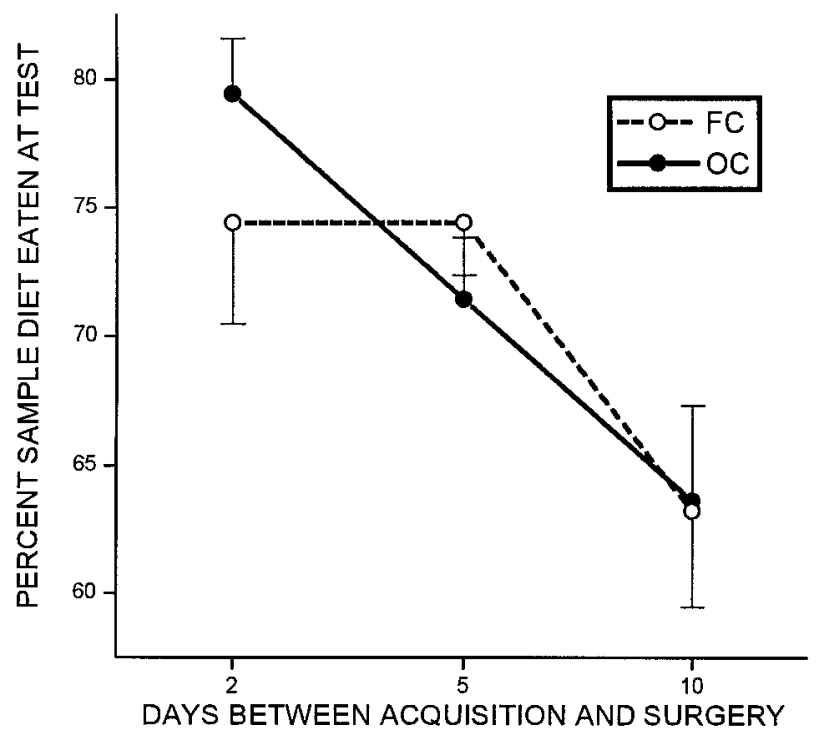

Figure 1. The amount of sample diet consumed by FC and OC groups, expressed as the mean percentage of the total amount of food consumed at the various delays in the (two-choice) retrograde amnesia test of Experiment 1 .

\section{Retrograde memory test}

The results for the retrograde memory test of Experiment 1 are presented in Figure 1 as the amount of the sample food consumed at test, expressed as a percentage of the total amount of food consumed at test. Both groups' preference for the sample food was greatest at the shortest ( $2 \mathrm{~d}$ ) delay and declined progressively thereafter. ANOVA applied to the data revealed a significant effect of delay, $F_{(2,24)}=5.94 ; p<0.001$, but neither the group, $F<1$, nor the group $\times$ delay interaction, $F<1$, was statistically significant.

\section{Anterograde memory test}

The results for the anterograde memory test of Experiment 1 are presented in Figure 2. The FC and OC groups exhibited strongest preference for the sample food when tested immediately after the $\mathrm{D}-\mathrm{S}$ interaction. Although the OC groups ate slightly more of the sample food than the FC groups over the various delay intervals, the overall difference was not statistically significant. ANOVA confirmed that the main effect of group, $F_{(1,24)}=3.07 ; p>0.05$, and the group $\times$ delay interactions, $F<1$, were not significant. As in the retrograde memory test, the only significant effect was that of delay, $F_{(2,24)}=6.95 ; p<0.004$.

\section{EXPERIMENT 2}

In Experiment 1, frontal cortex lesions did not affect rats' acquisition and long-term retention of a socially transmitted food preference (anterograde memory) or retrieval of the preference when it was acquired preoperatively (retrograde memory). It is possible that the two-choice task of Experiment 1 did not yield a lesion effect because rats could perform successfully without depending on strategic operations that required involvement of the frontal cortex. In a study of age differences in nonmatchingto-sample learning, a test known to assess frontal cortex function, Gagnon and Winocur (1995) found that age-related deficits were substantially greater when the number of choices at test was increased from two to three. Guided by these results, for Experiment 2, the task was changed so that the rats must select the

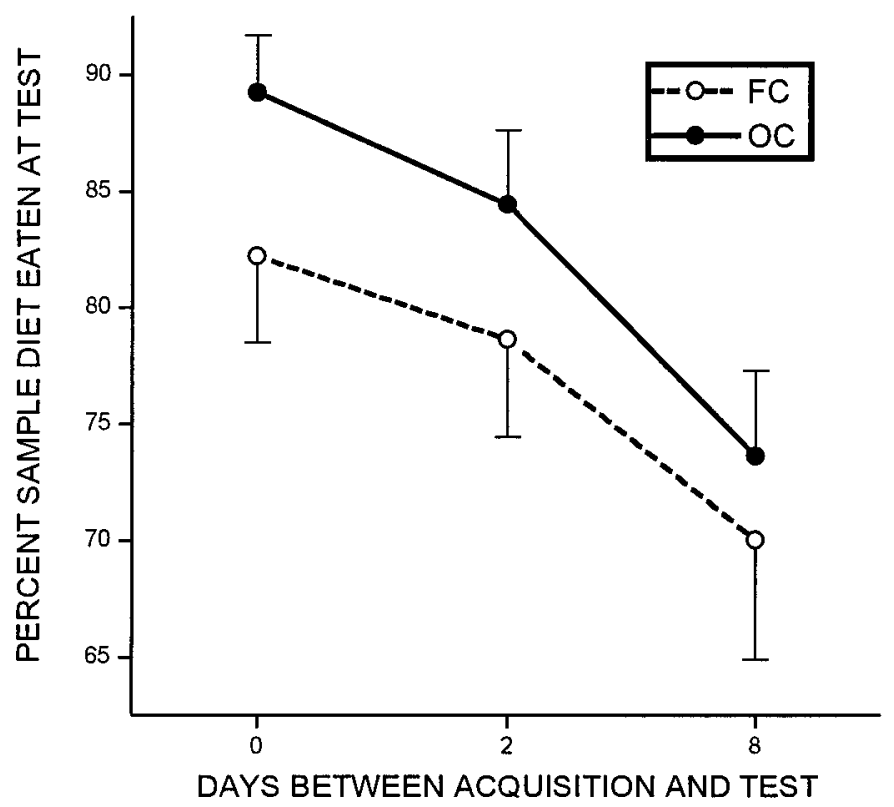

Figure 2. The amount of sample diet consumed by FC and OC groups, expressed as the mean percentage of the total amount of food consumed at the various delays in the (two-choice) anterograde amnesia test of Experiment 1.

sample food from three equally desirable diets. It was reasoned that, in a three-choice situation, the rats would have to make a more directed and effortful search to identify the familiar (and safest) food. It was predicted that, under these relatively highinterference conditions, the performance of frontal cortexdamaged rats would suffer, particularly in the retrograde memory test, where longer delays between food-preference training and test would reduce the distinctiveness of the sample food, and put increased pressure on response-selection processes.

\section{MATERIALS AND METHODS}

\section{General procedure}

Sixty-four male, Long-Evans rats served as Ss in the retrograde memory condition. After food-preference training, $32 \mathrm{Ss}$ received frontal cortex lesions, and the other 32 underwent control surgery, according to procedures described in Experiment 1 . After a 10 d recovery period, S rats' food preferences were assessed in a three-choice test (see retrograde memory condition below for details). Several weeks later, after Ss participated in a contextual conditioning test, the anterograde memory test was initiated (see anterograde memory condition below for details). During the intervening period, two OC, S rats died so that only 30 OC rats were available for the anterograde memory test. A total of 10 rats served as Ds for the retrograde and anterograde memory tests.

After anterograde memory testing, rats in the FC group were killed, their brains removed, and their lesions examined histologically as described above.

Retrograde memory test. In Experiment 2, there were two notable departures from the preoperative training procedures of the retrograde memory test followed in Experiment 1. First, the D rats ate from one of three sample diets, which consisted of powdered chow mixed with cocoa ( $2 \%$ by weight), ground cinnamon ( $1 \%$ by weight), or ground ginger $(1 \%$ by weight). Preliminary investigations showed that, at these concentrations, normal adult rats were equally likely to eat from the three diets. In the second departure, a $1 \mathrm{~d}$ delay condition between $\mathrm{D}-\mathrm{S}$ interaction and surgery was introduced to produce a finer measure of temporally graded RA. Postoperative retrograde memory testing was the same as in Experiment 1 except that, in Experiment 2, S rats were presented with the sample diet along with the other two diets. The spatial arrangement of the diets was varied according to a randomly determined order.

Anterograde memory test. As in Experiment 1, S rats were randomly assigned to the delay conditions of the anterograde memory test. In 


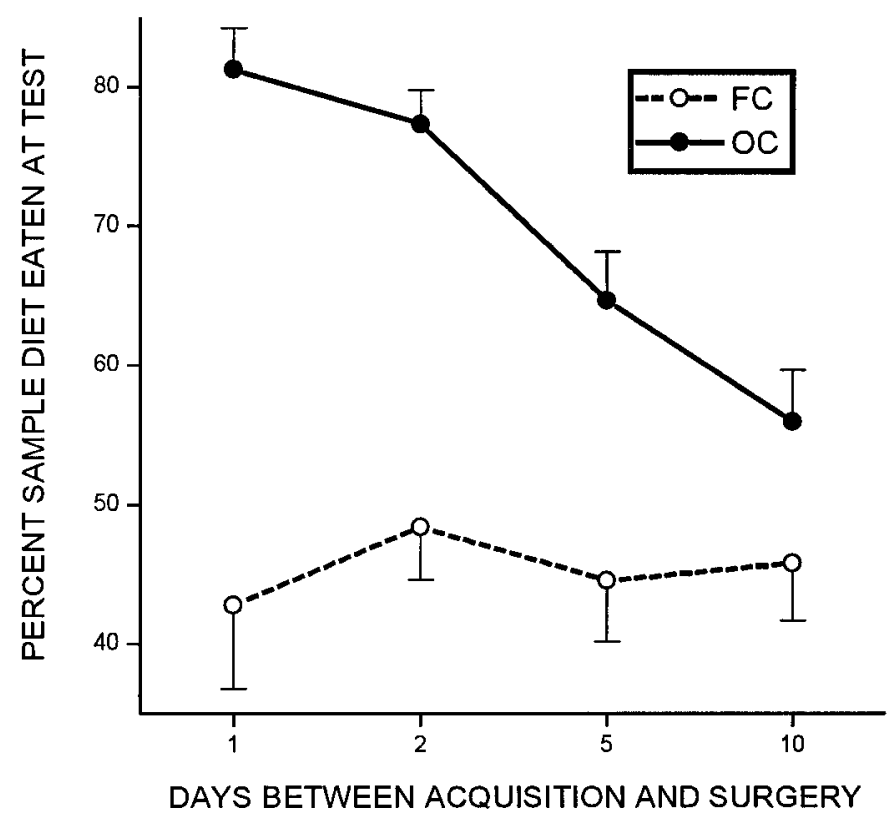

Figure 3. The amount of sample diet consumed by FC and OC groups, expressed as a percentage of the total amount of food consumed at the various delays in the (three-choice) retrograde amnesia test of Experiment 2 .

Experiment 2, there were five delays between $\mathrm{D}-\mathrm{S}$ interaction and testing: $0,1,2,4$, or $8 \mathrm{~d}$. The only other procedural difference from the anterograde memory test of Experiment 1 was that $\mathrm{D}$ rats were fed one of three sample diets (cocoa, cinnamon, or ginger), and all three diets were available at test.

\section{RESULTS}

The type of diet used as sample did not affect the results of either group in the anterograde and retrograde memory tests. ANOVA revealed nonsignificant main effects of diet and nonsignificant diet $\times$ group interactions (all $p$ values $>0.05$ ). Once again, the sample diets were combined for purposes of data presentation. Because there were no group differences related to when, in the test sessions, food preferences were measured, only the $2 \mathrm{hr}$ scores will be presented.

\section{Retrograde memory test}

As can be seen in Figure 3, there was a dramatic effect of lesion on food preference in the retrograde memory test. The OC rats, as expected, showed strongest memory for the food preference when it was acquired most recently, with declining performance at longer delays. By comparison, the FC groups showed no gradient over the delay periods and, at no time, ate more than an average of $50 \%$ of the sample diet. Although above the $33 \%$ chance level, this was considerably below that consumed by the OC groups at all delays.

These observations were confirmed by ANOVA, which revealed significant main effects of group, $F_{(1,56)}=74.51, p<$ 0.0001 ; delay, $F_{(3,56)}=4.25, p<0.01$; and a significant group $\times$ delay interaction, $F_{(3,56)}=4.60, p<0.006$. Post hoc analyses with the Tukey test indicated highly significant group differences at 1 , 2 , and $5 \mathrm{~d}$ delays $(p$ values $<0.01$ ) but not at the $10 \mathrm{~d}$ delay.

Because the scores for the two groups were similar at the longest delay, the data may be seen as reflecting a temporally graded deficit in the FC group. However, an examination of eating patterns at the various intervals revealed a gradient only in the OC group. At each interval, the amount of the sample food

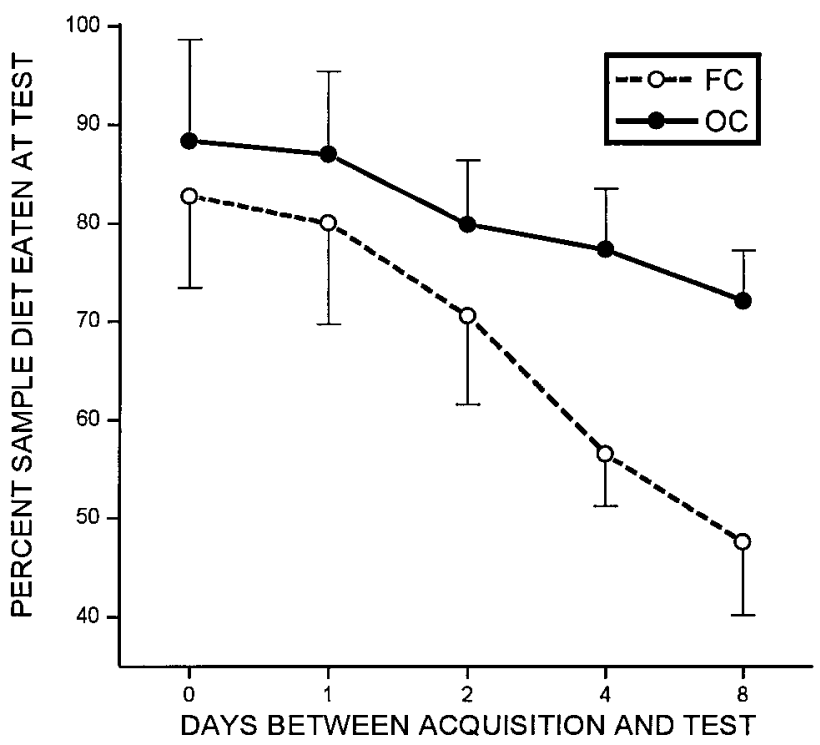

Figure 4. The amount of sample diet consumed by FC and OC groups, expressed as a percentage of the total amount of food consumed at the various delays of the (three-choice) anterograde amnesia test of Experiment 2 .

eaten by the FC group during the test was $\sim 45 \%$ of the total food eaten, and this value hardly varied at the different intervals. A comparison of the scores of the FC group at the shortest $(42.8 \%)$ and longest (45.8\%) delays revealed a percentage increase of only $12.5 \%$, a difference that did not approach statistical significance $(t<1)$. Over the same period, the preference of the OC group for the sample food declined by $31.2 \%(81.3-55.9 \%)$, a highly significant difference, $t_{(7)}=4.68, p<0.002$.

\section{Anterograde memory test}

The results of the anterograde memory test are presented in Figure 4. As can be seen, the OC groups displayed the expected time-related decline in preference for the sample food. The FC group also showed declining memory for the food preference with increased delays. Between 0 and $2 \mathrm{~d}$ there was little difference in performance between the lesion groups but beyond the $2 \mathrm{~d}$ delay, the FC group exhibited a faster rate of forgetting.

These observations were confirmed by ANOVA, which revealed significant main effects of group, $F_{(1,52)}=43.34, p<$ 0.0001 ; delay, $F_{(4,52)}=23.407, p<0.0001$; and a significant group $\times$ delay interaction, $F_{(4,52)}=3.60, p<0.02$. Post hoc analysis with the Tukey test showed that the significant interaction was caused by significant group differences in the 4 and $8 \mathrm{~d}$ delay conditions $(p$ values $<0.01)$.

\section{Relationship between $A A$ and $R A$}

With respect to the controversy over the relationship between AA and $\mathrm{RA}$, the present results suggest that, in the case of frontal cortex-damaged rats, AA at long delays was related to retrograde memory loss. An overall Pearson product correlational analysis, that compared the performance of each frontal cortex-damaged rat in the AA and RA tests, did not reveal a significant correlation, $r=0.07$. On the other hand, the same analysis, performed only on the scores of FC groups in the 4 and $8 \mathrm{~d}$ conditions of the AA test, where there were clear signs of anterograde memory loss, with their corresponding scores in the RA test, did yield a significant correlation, $r=0.58, p<0.05$. 


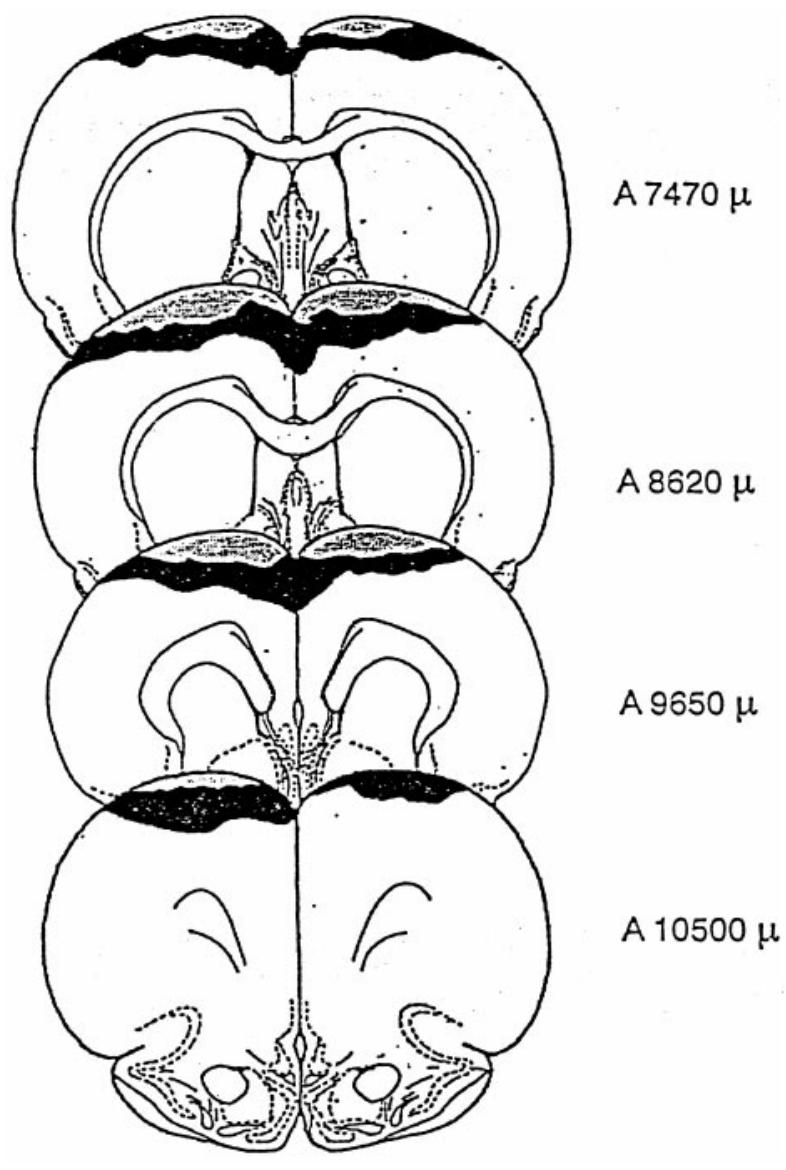

Figure 5. Maximal and minimal extents of frontal cortex lesions.

\section{Histological report}

Maximal and minimal extents of frontal cortex lesions are represented diagramatically in Figure 5. The lesions extended between 7.5 and $10.5 \mathrm{~mm}$ anterior to the interaural plane, the greatest amount of cortical damage occurring between 8.5 and $9.7 \mathrm{~mm}$. The lesions were comparable to those reported in previous work (Winocur, 1992; Winocur and Eskes, 1998). The region of the frontal cortex that sustained the most damage was the dorsomedial shoulder of the frontal cortex (Fr2) or premotor area. In most cases, the lesions also extended posteriorly into dorsolateral areas and, in a few rats with the largest lesions, there was slight damage to anterior cingulate. There were no substantial differences between the frontal cortex lesions in rats of Experiments 1 and 2, nor was there a relationship between size or predominant location of lesion and the behavioral results.

\section{DISCUSSION}

The present study assessed remote memory after damage to frontal cortex in a rat model, using a paradigm that provided evidence of temporally graded RA in rats with hippocampal lesions (Winocur, 1990). In Experiment 1, FC and OC groups preoperatively acquired a food preference at different times and then, postoperatively, were presented with the sample food along with an unfamiliar food. There was no effect of lesion, because both groups exhibited normal memory for the preferred food. A similar procedure was followed in Experiment 2 except that, at test, rats were confronted with a three-choice recognition test that consisted of the sample food plus two unfamiliar foods. This manipulation had the dramatic effect of virtually eliminating the lesioned rats' ability to express preoperatively acquired food preferences.

These results show that, under certain conditions, damage to frontal cortex of rats can affect remote memory function but that the deficit differs from the RA associated with hippocampal damage. For example, in the two-choice RA test, in which hippocampal rats exhibited temporally graded RA (Winocur, 1990), FC groups performed normally. Clearly, the frontal cortex is not essential for mediating the basic processes of acquiring and retaining food preferences. However, when in Experiment 2, response selection was made more difficult by an additional distractor, rats with frontal lesions experienced severe RA. Moreover, when frontal cortex lesions did produce remote memory impairment, the effect was constant over all delay intervals.

The poor performance of FC groups on the three-choice test was undoubtedly related to the increased demands created by the third food option, as it was in Gagnon and Winocur's (1995) study of aged rats. In a study that bears even more directly on the present findings, Winocur and Eskes (1998) tested conditional associative learning in rats with identical frontal cortex lesions. FC groups performed at near normal levels when the correct response contrasted from other possibilities, but were severely impaired when response alternatives were similar. These results, along with the present findings, indicate that rats with frontal cortex impairment are vulnerable to manipulations that involve searching for the proper cue, discriminating among similar cues, and selecting among similar response alternatives. As well, the present results highlight the important point that even recognition memory, which typically resists the effects of frontal damage (Janowsky et al., 1989; Milner et al., 1991) can be compromised by increasing the number of response alternatives and their similarity. The overall pattern is consistent with a substantial neuropsychological literature on humans that implicates the frontal lobes in strategic cognitive function and strategic retrieval in memory tests, in particular (for review, see Stuss et al., 1994; Shimamura, 1994; Moscovitch and Winocur, 1995; and recent studies by Ergis et al., 1997; Thompson-Schill et al., 1997).

In the present study, the selective difficulty of the FC groups in the three-choice test of retrograde memory was also reflected in the test of anterograde memory. However, whereas the temporal gradient of the RA of the FC groups was flat, their AA revealed a time-related gradient. In the AA tests, rats with frontal cortex lesions performed normally at short delays but, at longer delays, their performance deteriorated. The latter finding is significant for several reasons. First, it shows that the flat gradient of the FC group in the retrograde memory test was not simply a function of the three-choice test. Second, this pattern shows that the vulnerability of frontal cortex-damaged rats to high-interference tasks extends to anterograde memory. Third, the AA data show that the length of the interval between acquisition and test contributes to memory deficits associated with damage to frontal cortex. In the three-choice anterograde test, the FC group was not impaired until 2-4 d had elapsed since acquisition. In the retrograde memory test, where lesion-induced deficits were observed at all delays, the shortest interval between acquisition and testing (including the postoperative recovery period), was $12 \mathrm{~d}$. Considered together, these results indicate that the ability of frontal cortexdamaged rats to perform successfully in memory tests is affected by various factors, e.g., level of interference and elapsed time, that add to the strategic demands of the task.

It is noteworthy that, in the RA test of Experiment 2, the 
memory of the FC group for the acquired food preference differed significantly from that of the OC group when the interval between the D-S interaction and surgery was 1,2 , or $5 \mathrm{~d}$, but not at the $10 \mathrm{~d}$ interval. The convergence of scores in the $10 \mathrm{~d}$ condition is related to the declining preference of the control groups for the sample food, which is typical of normal rats in this task (Galef and Wigmore, 1983; Winocur, 1990), and not to a reduction in the deficit of the FC group, which might suggest a temporally graded RA. Indeed, the temporal gradient for the FC group was virtually flat. Clearly, the similarity in the test scores of the groups at the $10 \mathrm{~d}$ interval was attributable to the progressive memory loss of the OC groups, rather than memory recovery in the FC groups. The flat gradient of the FC groups can be contrasted with the sloped function observed previously in rats with hippocampal lesions (Winocur, 1990). In the latter experiment, hippocampal rats displayed better memory at long intervals than short intervals, thereby demonstrating a true temporally graded RA.

An important finding of the present research was that AA at long retention intervals and RA were correlated in rats with frontal cortex damage. This result was undoubtedly related to the fact that both tests were similar in format and in terms of their demands on memory processes. Consequently, it is unlikely that other cognitive processes, mediated by frontal cortex, contributed differentially to performance on the two tests in a way that could have obscured overlapping features of the respective deficits. The relationship between AA and RA is not fully understood, and evidence from some clinical populations (Korsakoff Syndrome) that the two are at least partially independent (Kopelman, 1989), has led some investigators to suggest that RA may represent a "distinct neurological entity" (Kapur, 1993; see also Levine et al., 1998). On the other hand, the present results and those of others, show that certain types of brain damage can produce cognitive deficits that affect both anterograde and retrograde memory functions. Thus, hippocampal lesions that affect consolidation processes lead to severe anterograde amnesia and frequently to a temporally graded retrograde amnesia in animals and humans (Winocur, 1990; Kim and Fanselow, 1992; Rempel-Clower et al., 1996; Reed and Squire, 1998). Similarly, in humans, frontal lobe lesions, which disrupt strategic retrieval processes can lead to retrograde amnesia with a flat gradient, and anterograde amnesia at long intervals between acquisition and retrieval (Della Sala et al., 1993; Mangels et al., 1996; Levine et al., 1998), in ways that complement the present results.

Among the various theories of frontal lobe function, it has been proposed that the structure is centrally involved in working memory, the process of retaining information while performing other tasks (Goldman-Rakic, 1987). Moscovitch and Winocur (1992) have advanced a working-with-memory hypothesis that incorporates the basic working memory notion, as well as the role of the frontal lobes role in strategic planning and inhibitory control. According to this view, the frontal lobes are not involved in the straightforward recovery of accessible information; that function is associated with medial-temporal lobe/diencephalic mechanisms. Rather, the frontal lobes work with memory to direct goal-oriented strategies and achieve specific objectives. For example, in new learning the frontal lobes serve to organize information into meaningful units that can be easily processed and retained. At retrieval, in the absence of useful external cues, the frontal lobes initiate appropriate search operations aimed at finding targeted memory traces and placing them into appropriate spatial/temporal context. In tasks that involve matching and comparisons (e.g., matching- or non-matching-to-sample learn- ing), the frontal lobes integrate information from past experience with current task requirements for purposes of response selection and planning. Factors such as the passage of time, the number of competing associations, and difficulty in placing recalled information in spatial/temporal context, add to the complexity of this process and create additional demands on the frontal lobes. The food-preference task, which requires rats to select a specific diet from among several alternatives, on the basis of past experience, incorporates all of the above factors.

Finally, there is controversy over whether certain frontal regions associated with strategic functions in primates exist in homologous form in the rat (for review, see Uylings and van Eden, 1990; Preuss, 1995). For example, in primates, the frontal area most frequently identified with strategic processes that support information retrieval is the dorsolateral frontal cortex (DLFC). Traditionally, the ventromedial frontal cortex has been identified as the corresponding area in the rat brain (Kolb, 1990). However, on anatomical evidence, Preuss (1995) refutes this connection and, indeed, makes the convincing argument that the dorsolateral frontal cortex does not have a homolog in the rat brain.

The present study was not designed to address this issue, but aspects of the results are relevant. Our lesions affected primarily the dorsomedial shoulder of the frontal cortex (Fr2) and posterior dorsolateral regions that together define the premotor cortex in the rat brain. For the most part, the ventromedial frontal cortex was spared, suggesting, in line with Preuss' position, that this region is not sufficient to support those cognitive processes that are essential for retrieving stored information. The results also underscore the importance of distinguishing between structural and functional homologs. They provide clear evidence that damage to other frontal regions in the rat (e.g., premotor cortex) can disrupt performance on strategic tasks that require working-withmemory functions that are mediated by DLFC in monkeys and humans. The premotor cortex is not necessarily structurally homologous with the DLFC in monkeys and humans, but our findings suggest that they perform comparable functions. This notion, if supported by future research, will force a reconsideration of the functional organization of the rat's frontal cortex, and will have important implications for attempts to develop a rat model of frontal lobe function in higher mammals.

\section{REFERENCES}

Cho YH, Kesner BP, Brodale S (1995) Retrograde and anterograde amnesia for spatial discrimination in rats: role of hippocampus, entorhinal cortex, and parietal cortex. Psychobiology 23:185-194.

Della Sala S, Laiacona M, Spinnler H, Trivelli C (1993) Autobiographical recollection and frontal damage. Neuropsychologia 31:823-839.

Ergis AM, Winocur G, Saint-Cyr J, Van der Linden M, Melo B, Freedman M (1997) Impaired recognition in Parkinson's disease. Brain Cog 3:310-312.

Gagnon S, Winocur G (1995) A comparison of old and young rats' performance on a test of nonmatching-to-sample. An analysis of agerelated encoding and memory deficits. Psychobiology 23:322-328.

Galef Jr BG, Wigmore SR (1983) Transfer of information concerning distant foods: A laboratory investigation of the "information centre" hypothesis. Anim Behav 31:748-758.

Goldman-Rakic PS (1987) Circuitry of primates prefrontal cortex and regulation of behaviour by representational memory. In: Handbook of Physiology: the nervous System, Vol 5 (Plum F, Mountcastle U, eds), pp 373-417. Bethesda, MD: American Physiological Society.

Janowsky JS, Shimamura AP, Squire LR (1989) Source memory impairment in patients with frontal lobe lesions. Neuropsychologia 27:1043-1056.

Kapur N (1993) Focal retrograde amnesia: a critical review. Cortex 29:217-234. 
Kim JJ, Fanselow MS (1992) Modality-specific retrograde amnesia of fear. Science 256:675-677.

Kolb B (1990) Animal models for human PFC-related disorders. Prog Brain Res 85:501-519.

König JR, Klippel RE (1963) The rat brain: a stereotaxic atlas of the forebrain and lower parts of the brain stem. Baltimore: Williams and Wilkins.

Kopelman MD (1989) Remote and autobiographical memory, temporal context memory and frontal atrophy in Korsakoff and Alzheimer patients. Neuropsychologia 27:437-460.

Levine B, Black SE, Cabeza R, Sinden M, Mcintosh AR, Toth JP, Tulving E, Stuss DT (1998) Episodic memory and the self in a case of isolated retrograde amnesia. Brain 121:1951-1973.

Mangels JA, Gershberg FB, Knight RT, Shimamura AP (1996) Impaired retrieval from remote memory in patients with frontal lobe damage. Neuropsychology 10:32-41.

Marslen-Wilson W, Teuber H-L (1975) Memory for remote events in retrograde amnesia. Neuropsychologia 13:353-364.

Milner B, Corsi P, Leonard G (1991) Frontal-lobe contribution to recency judgments. Neuropsychologia 29:601-618.

Moscovitch M (1994) Cognitive resources and dual-task interference effects at retrieval in normal people: the role of the frontal lobes and medial temporal cortex. Neuropsychology 8:524-534.

Moscovitch M, Winocur G (1992) The neuropsychology of memory and aging. In: The handbook of aging and cognition. (Craik FIM, Salthouse TA, eds), pp 315-372. Hillsdale, NJ: Erlbaum.

Moscovitch M, Winocur G (1995) Frontal lobes, memory, and aging. In: Structure and functions of the human prefrontal cortex (Grafman J, Holyoak KJ, Boller B, eds), pp 119-150. New York: The New York Academy of Sciences.

Munsey M, Eichenbaum H (1995) Selective damage to the hippocampal region blocks long-term retention of a natural and nonspatial stimulusstimulus association. Hippocampus 5:546-556.

Nadel L, Moscovitch M (1997) Memory consolidation, retrograde amnesia and the hippocampal complex. Curr Opin Neurobiol 7:217-227.

Preuss TM (1995) Do rats have prefrontal cortex? The Rose-WooleyAkert program reconsidered. J Cognit Neurosci 7:1-24.

Reed JM, Squire LR (1998) Retrograde amnesia for facts and events: Findings from four new cases. J Neurosci 18:3493-3954.

Rempel-Clower N, Zola SM, Squire LR, Amaral DG (1996) Three cases of enduring memory impairment following bilateral damage limited to the hippocampal formation. J Neurosci 16:5233-5255.

Schmidtke K, Vollmer H (1997) Retrograde amnesia: a study of its relation to anterograde amnesia and semantic memory deficits. Neuropsychologia 35:505-518.

Shimamura AP (1994) Memory and frontal lobe function. In: The cognitive neurosciences (Gazzaniga MS, ed), pp 803-813. Cambridge: MIT.

Shimamura AP, Squire LR (1986) Korsakoff's syndrome: a study of the relation between anterograde amnesia and remote memory impairment. Behav Neurosci 100:165-170.

Stuss DT, Alexander MP, Palumbo CL, Buckle L, Sayer L, Pogue J (1994) Organizational strategies of patients with unilateral or bilateral frontal lobe injury in word list learning tasks. Neuropsychology 8:355-373.

Thompson-Schill SL, D'Esposito M, Aguirre GK, Farah MJ (1997) Role of left inferior prefrontal cortex in retrieval of semantic knowledge: a reevaluation. Proc Natl Acad Sci USA 94:14792-14797.

Uylings HBM, van Eden CG (1990) Qualitative and quantitative comparison of the prefrontal cortex in rat and in primates, including humans. Prog Brain Res 85:31-62.

Wheeler MA, Stuss DT, Tulving E (1995) Frontal lobe damage produces episodic memory impairment. J Int Neuropsychol Soc 1:525-536.

Wheeler MA, Stuss DT, Tulving E (1997) Toward a theory of episodic memory: the frontal lobes and autonoetic consciousness. Psychol Bull 121:331-354.

Winocur G (1990) Anterograde and retrograde amnesia in rats with dorsal hippocampal or dorsomedial thalamic lesions. Behav Brain Res $38: 145-154$.

Winocur G (1992) The hippocampus and prefrontal cortex in learning and memory: an animal model approach. In: Neuropsychology of memory (Squire LR, Butters N, eds), pp 429-439. New York: Guilford.

Winocur G, Eskes G (1998) Prefrontal cortex and caudate nucleus in conditional associative learning: dissociated effects of selective brain lesions. Behav Neurosci 112:1-14.

Winocur G, Oxbury S, Roberts R, Agnetti V, Davis C (1984) Amnesia in a patient with bilateral lesions to the thalamus. Neuropsychologia 22:123-143.

Zola-Morgan S, Squire LR (1990) The primate hippocampal formation: evidence for a time-limited role in memory storage. Science 250:288290. 